\title{
STRONG LAWS OF LARGE NUMBERS FOR WEIGHTED SUMS OF RANDOM ELEMENTS IN NORMED LINEAR SPACES
}

\author{
ANDRE ADLER \\ Department of Mathematics \\ Illinois Institute of Technology \\ Chicago, Illinois 60616 U.S.A. \\ ANDREW ROSALSKY \\ Department of Statistics \\ University of Florida \\ Gainesville, Florida 32611 U.S.A. \\ ROBERT L. TAYLOR \\ Department of Statistics \\ University of Georgia \\ Athens, Georgia 30602 U.S.A. \\ (Received December 16, 1988 and in revised form February 20, 1989)
}

ABSTRACT. Consider a sequence of independent random elements $\left(V_{n}, n \geq 1\right\}$ in a real separable normed linear space $X$ (assumed to be a Banach space in most of the results), and sequences of constants $\left\{a_{n}, n \geq 1\right\}$ and $\left\{b_{n}, n \geq 1\right\}$ with $0<b_{n} \uparrow \infty$. Sets of conditions are provided for $\left\{a_{n}\left(V_{n}-E V_{n}\right), n \geq 1\right\}$ to obey a general strong law of large numbers of the form $\sum_{j=1}^{n} a_{j}\left(V_{j}-E V_{j}\right) / b_{n} \rightarrow 0$ almost certainly. The hypotheses involve the distributions of the $\left\{V_{n}, n \geq 1\right\}$, the growth behaviors of $\left\{a_{n}, n \geq 1\right\}$ and $\left\{b_{n}, n \geq 1\right\}$, and for some of the results impose a geometric condition on $X$. Moreover, Feller's classical result generalizing the Marcinkiewicz-Zygmund strong law of large numbers is shown to hold for random elements in a real separable Rademacher type $p(1<\mathrm{p} \leq 2)$ Banach space.

KEY WORDS AND PHRASES. Real separable Banach space, independent random elements. normed weighted sums, strong law of large numbers, almost certain convergence, stochastically dominated random elements, Rademacher type p, Beck-convex normed linear space, Schauder basis. (uniformly) tight sequence. 


\section{INTRODUCTION.}

Let $(\Omega, F, P)$ be a probability space and let $X$ be a real separable normed linear space with norm $|1 \cdot| 1$. It is supposed that $X$ is equipped with its Borel $\sigma$-algebra $B$. That is, $B$ is the $\sigma$-algebra generated by the class of open subsets of $X$ determined by $11 \cdot 11$. A random element $V$ in $X$ is an $F$-measurable transformation from $\Omega$ to the measurable space $(X, B)$. The expected value of $V$, denoted EV, is defined to be the Pettis integral provided it exists. That is, $\mathrm{V}$ has expected value $E V \in X$ if $f(E V)=E(f(V))$ for every $f \in X^{*}$ where $X^{*}$ denotes the (dual) space of all continuous linear functionals on $X$. The definitions of independence and identically distributed for random elements are similar to those in the (real-valued) random variable case.

Consider a sequence of independent random elements $\left\{V_{n}, n \geq 1\right\}$, all of whose expected values exist. Let $\left\{a_{n}, n \geq 1\right\}$ and $\left\{b_{n}, n \geq 1\right\}$ be constants with $0<b_{n} \uparrow \infty$. Then $\left\{a_{n}\left(V_{n}-E V_{n}\right), n \geq 1\right\}$ is said to obey the general strong law of large numbers (SLLN) with norming constants $\left(b_{n}, n \geq 1\right\}$ if the normed weighted sum $\sum_{j=1}^{n} a_{j}\left(V_{j}-E V_{j}\right) / b_{n}$ converges almost certainly to the zero element in $X$ (denoted by 0 ), and this will be written

$$
\frac{\sum_{j=1}^{n} a_{j}\left(V_{j}-E V_{j}\right)}{b_{n}} \rightarrow 0 \text { a.c. }
$$

Herein, the main results furnish conditions on $X$, on the distributions of the $\left\{V_{n}, n \geq 1\right\}$, and on the growth behavior of the constants $\left\{a_{n}, n \geq 1\right\}$ and $\left\{b_{n}, n \geq 1\right\}$ which ensure that the SLLN (1.1) obtains. In most of the results $X$ is assumed to be a Banach space, and in many of the results $\left\{V_{n}, n \geq 1\right\}$ is assumed to be stochastically dominated by a random element $V$ in the sense that for some constant $D<\infty$

$$
\left.P|| \mid V_{n} \|>t\right\} \leq D P\{\mid D V \|>t\}, \quad t \geq 0, n \geq 1 \text {. }
$$

Of course, (1.2) is automatic with $V=V_{1}$ and $D=1$ if the $\left(V_{n}, n \geq 1\right)$ are independent and identically distributed (i.i.d.) and even in this case the results are new. In Section 3, SLLN's are established under geometric conditions on $X$ whereas in Section 4, SLLN's are established without such conditions. The SLLN problem was studied by Adler and Rosalsky $[1,2]$ in the random variable case, and some of those results will be extended to the random element case in the current work.

Taylor [3] provided a comprehensive and unified treatment of results under whose conditions $\sum_{j=1}^{n} a_{n j} V_{j} \rightarrow 0$ a.c. where $\left\{V_{n}, n \geq 1\right\}$ are independent, mean zero random elements in a real separable normed linear space and $\left\{a_{n j}, 1 \leq j \leq n, n \geq 1\right\}$ is a triangular array of constants. Some of the 
arguments in Taylor's monograph utilized a result of Rohatgi [4] which will now be stated. (Rohatgi's work generalized earlier work of Pruitt [5].)

THEOREM (Rohatgi [4]). Let $\left(X_{n}, n \geq 1\right\}$ be independent, mean zero random variables and let $X$ be an $L_{p}$ random variable for some $p>1$. Suppose that $\left(X_{n}, n \geq 1\right)$ is stochastically dominated by $X$ in the sense that

$$
P\left(\left|X_{n}\right|>t\right\} \leq P(|X|>t), \quad t \geq 0, n \geq 1 .
$$

Let $\left\{a_{n j}, 1 \leq j \leq n, n \geq 1\right\}$ be constants satisfying $\lim _{n \rightarrow \infty} a_{n j}=0$ for each $j \geq 1, \sum_{j=1}^{n}\left|a_{n j}\right|=O(1)$, and

$$
\max _{1 \leq j \leq n}\left|a_{n j}\right|=O\left(n^{-1 /(p-1)}\right)
$$

Then $\sum_{j=1}^{n} a_{n j} X_{j} \rightarrow 0$ a.c.

In Theorems 8 and 9 and Corollary 2 of the current work, versions of some of the results presented in Taylor [3] will be obtained under less restrictive conditions but only for the case where $a_{n j}=a_{j} / b_{n}, 1 \leq j \leq n, n \geq 1$, where $\left\{a_{n}, n \geq 1\right\}$ and $\left\{b_{n}, n \geq 1\right\}$ are constants. The arguments will not involve Rohatgi's theorem but, rather, will employ Corollary 1 below. Corollary 1 plays a role in the proofs similar to the role that Rohatgi's theorem played in establishing the counterparts presented in Taylor [3]. Corollary 1 has less stringent conditions than Rohatgi's theorem when $a_{n j}=a_{j} / b_{n}, 1 \leq j \leq n, n \geq 1$. Specifically, if that choice of $\left\{a_{n j}, 1 \leq j \leq n, n \geq 1\right\}$ satisfies (1.3), then

$$
\frac{a_{n}}{b_{n}}=O\left(n^{-1 /(p-1)}\right)
$$

which is stronger than the condition

$$
\frac{a_{n}}{b_{n}}=O\left(n^{-1 / p}\right)
$$

of Corollary 1. Thus, if $\left\{a_{n j}, 1 \leq j \leq n, n \geq 1\right\}$ satisfies $b_{n} / a_{n}=O\left(n^{\alpha}\right)$ for some $1 / 2<\alpha \leq 1$, then to invoke Rohatgi's theorem requires that (1.4) and the moment condition $\mathrm{E}|\mathrm{X}|^{\mathrm{P}}<\infty$ hold where $p \geq 1+\frac{1}{\alpha} \geq 2$, whereas to invoke Corollary 1 merely requires that (1.5) and the (weaker) moment condition $\mathrm{E}|\mathrm{X}|^{\mathrm{P}}<\infty$ hold where $2>\mathrm{p} \geq \frac{1}{\alpha} \geq 1$. For example, for i.i.d. random variables $\left\{X_{n}, n \geq 1\right\}$ with $\operatorname{EX}_{1}=0$, the classical Kolmogorov SLLN $\sum_{j=1}^{n} X_{j} / n \rightarrow 0$ a.c. follows from Corollary 1 but not from Rohatgi's theorem (which would require $\operatorname{EX}_{1}^{2}<\infty$ ).

A SLLN for normed weighted sums of random elements in a real separable Banach space has been proved by Mikosch and Norvaiša [6], but their result and the current ones do not entail each other. 
For random elements in a real separable Banach space, the study of the SLLN problem dates back to the pioneering work by Mourier [7] (see also Laha and Rohatgi [8, p. 452] or Taylor [3, p. 72]) wherein a direct analogue of the classical Kolmogorov SLLN was established. More precisely, Mourier showed that if $\left(V_{n}, n \geq 1\right\}$ are i.i.d. random elements in a real separable Banach space and if $E\left\|\mid V_{1}\right\|<\infty$, then $\sum_{j=1}^{n}\left(V_{j}-E V_{1}\right) / n \rightarrow 0$ a.c. (A new propf of Mourier's SLLN has recently been discovered by Cuesta and Matran [9].) For random variables, the Kolmogorov SLLN was generalized by the Marcinkiewicz-Zygmund SLLN (see, e.g., Chow and Teicher [10, p. 122]) which, in tum, was generalized by Feller [11]. A random element version of Feller's result is presented in Theorem 4 below wherein it is assumed that the Banach space is of Rademacher type $p(1<p \leq 2)$.

\section{PRELIMINARIES.}

Some definitions will be discussed and lemmas will be presented prior to establishing the main results.

Let $\left(Y_{n}, n \geq 1\right)$ be a Bernoulli sequence, that is, $\left(Y_{n}, n \geq 1\right\}$ are i.i.d. random variables with $P\left\{Y_{1}=1\right\}=P\left\{Y_{1}=-1\right\}=1 / 2$. Let $X$ be a real separable Banach space and let $x^{\infty}=x \times x \times x \times \cdots$ and define

$$
c(X)=\left\{\left(v_{n}, n \geq 1\right) \in X^{\infty}: \sum_{n=1}^{\infty} Y_{n} v_{n} \text { converges in probability }\right\} .
$$

Let $1 \leq p \leq 2$. Then $X$ is said to be of Rademacher type $p$ if there exists a constant $0<C<\infty$ such that

$$
E|| \sum_{n=1}^{\infty} Y_{n} v_{n}||^{P} \leq C \sum_{n=1}^{\infty}|| v_{n}||^{P}
$$

for all $\left\{v_{n}, n \geq 1\right\} \in C(X)$. Hoffmann-Jørgensen and Pisier [12] proved for $1 \leq p \leq 2$ that a real separable Banach space is of Rademacher type $\mathrm{p}$ iff there exists a constant $0<\mathrm{C}<\infty$ such that

$$
E|| \sum_{j=1}^{n} V_{j}||^{P} \leq C \sum_{j=1}^{n} E|| V_{i} \|^{p}
$$

for every finite collection $\left\{V_{1}, \ldots, V_{n}\right\}$ of independent random elements with $E V_{j}=0$, $E|| V_{j}|| P<\infty, \quad 1 \leq j \leq n$.

If a real separable Banach space is of Rademacher type $p$ for some $1<p \leq 2$, then it is of Rademacher type $\mathrm{q}$ for all $1 \leq \mathrm{q}<\mathrm{p}$. Every real separable Banach space is of Rademacher type (at least) 1 while the $L_{p}$-spaces and $\ell_{p}$-spaces are of Rademacher type $\min \{2, p\}$ for $p \geq 1$. Every real separable Hilbert space and real separable finite-dimensional.Banach space is of Rademacher type 2.

A normed linear space $X$ is said to be $\underline{B e c k-c o n v e x}$ if there exists an integer $N \geq 1$ and a number $0<\varepsilon<1$ such that for all choices of $\left\{v_{1}, \ldots, v_{N}\right\} \subset X$ with ||$v_{j}|| \leq 1,1 \leq j \leq N$, 


$$
|| \pm v_{1} \pm \cdots \pm v_{N} \| \leq N(1-\varepsilon)
$$

for some choice of + and - signs. This property has been extensively studied by Giesy [13]. A real separable Banach space is Beck convex iff it is of Rademacher type $p$ for some $p>1$.

A Schauder basis for a normed linear space $X$ is a sequence $\left(\beta_{i}, i \geq 1\right\} \subset X$ such that for each $v \in X$ there exists a unique sequence of scalars $\left\{t_{i}, i \geq 1\right\}$ such that

$$
\lim _{\mathbf{m} \rightarrow \infty} \sum_{i=1}^{m} t_{i} \beta_{i}=v \text {. }
$$

A sequence of linear functionals $\left\{f_{i}, i \geq 1\right\}$ (called coordinate functionals for the basis $\left\{\beta_{i}, i \geq 1\right\}$ ) can be defined by letting $f_{i}(v)=t_{i}, i \geq 1$, where $v \in X$ and (2.1) holds, and a sequence of linear functions $\left\{U_{m}, m \geq 1\right\}$ (called partial sum operators for the basis $\left.\left(\beta_{i}, i \geq 1\right\}\right)$ can be defined by

$$
U_{m}(v)=\sum_{i=1}^{m} f_{i}(v) \beta_{i}, \quad v \in X, m \geq 1 .
$$

The residual operators $\left\{Q_{m}, m \geq 1\right\}$ are defined by

$$
\mathrm{Q}_{\mathrm{m}}(\mathrm{v})=\mathrm{v}-\mathrm{U}_{\mathrm{m}}(\mathrm{v}), \quad \mathrm{v} \in \mathrm{X}, \mathrm{m} \geq 1 .
$$

A Schauder basis is said to be a monotone basis if $\left\{1\left|U_{m}(v)\right| 1, m \geq 1\right\}$ is a monotone sequence for each $\mathrm{v} \in X$.

A sequence of random elements $\left\{V_{n}, n \geq 1\right\}$ in a normed linear space $X$ is said to be (uniformly) tight if for each $\varepsilon>0$, there exists a compact subset $K_{\varepsilon}$ of $X$ such that $P\left(V_{n} \in K_{\varepsilon}\right\} \geq 1-\varepsilon$ for all $n \geq 1$.

LEMMA 1 (Adler and Rosalsky [1]). Let $\mathrm{X}_{0}$ and $\mathrm{X}$ be random variables such that $\mathrm{X}_{0}$ is stochastically dominated by $\mathrm{X}$ in the sense that there exists a constant $\mathrm{D}<\infty$ such that

$$
P\left(\left|X_{0}\right|>t\right) \leq D P(|D X|>t), \quad t \geq 0 .
$$

Then for all $\mathrm{p}>0$

$$
\left.\mathrm{E}\left|\mathrm{X}_{0}\right| \mathrm{PI}\left(\left|\mathrm{X}_{0}\right| \leq t\right) \leq \mathrm{Dt}^{\mathrm{PP}}|| \mathrm{DX} \mid>\mathrm{t}\right\}+\mathrm{D}^{\mathrm{P}+1} \mathrm{E}|\mathrm{X}| \mathrm{P} I(|D X| \leq t), \quad t \geq 0
$$

LEMMA 2 (Adler and Rosalsky [1]). Let $\left\{\mathrm{X}_{\mathrm{n}}, \mathrm{n} \geq 1\right\}$ and $\mathrm{X}$ be random variables such that $\left\{X_{n}, n \geq 1\right\}$ is stochastically dominated by $X$ in the sense that there exists a constant $D<\infty$ such that

$$
P\left\{\left|X_{n}\right|>t\right\} \leq D P\{|D X|>t\}, \quad t \geq 0, n \geq 1 .
$$

Let $\left\{c_{n}, n \geq 1\right\}$ be positive constants such that $\left[\max _{1 \leq j \leq n} c_{j}^{p}\right] \sum_{j=n}^{\infty} \frac{1}{c_{j}^{p}}=O(n)$ for some $p>0$ and $\sum_{n=1}^{\infty} P\left\{|X|>D c_{n}\right\}<\infty$. Then for all $0<M<\infty$, 


$$
\sum_{n=1}^{\infty} \frac{1}{c_{n}^{p}} E\left|X_{n}\right|^{P} I\left(\left|X_{n}\right| \leq M c_{n}\right)<\infty
$$

LEMMA 3. Let $X_{0}$ and $X$ be random variables such that $X_{0}$ is stochastically dominated by $X$ in the sense that $(2.2)$ holds. Then

$$
E\left|X_{0}\right| I\left(\left|X_{0}\right|>x\right)=\int_{x}^{\infty} P\left\{\left|X_{0}\right|>t\right\} d t+x P\left(\left|X_{0}\right|>x\right\}, \quad x \geq 0
$$

and

$$
\mathrm{E}\left|\mathrm{X}_{0}\right| \mathrm{I}\left(\left|\mathrm{X}_{0}\right|>\mathrm{x}\right) \leq \mathrm{D}^{2} \mathrm{E}|\mathrm{X}| \mathrm{I}(|\mathrm{DX}|>\mathrm{x}), \quad \mathrm{x} \geq 0 .
$$

PROOF. Integration by parts yields (2.4), and then (2.5) follows immediately from (2.4) and (2.2)

LEMMA 4 (Adler and Rosalsky [2]). Let $\mathrm{X}$ be a random variable such that $\mathrm{P}\{|\mathrm{X}|>t)$ is regularly varying with exponent $\rho<-1$. Then $X \in L_{p}$ for all $0<p<-\rho$ and

$$
E|X| I(|X|>t)=(1+o(1))\left[\frac{\rho}{\rho+1}\right] t P(|X|>t) \text { as } t \rightarrow \infty \text {. }
$$

The next lemma shows that stochastic dominance can be accomplished by a sequence of random variables having a bounded absolute $p$-th moment $(p>1)$.

LEMMA 5 (Taylor $\left[3\right.$, p. 123]). Let $\left\{X_{n}, n \geq 1\right\}$ be random variables such that $\sup _{n \geq 1} E\left|X_{n}\right|^{p}<\infty$ for some $p>1$. Then there exists a random variable $X$ with $E|X|^{q}<\infty$ for all $0<\mathrm{q}<\mathrm{p}$ such that

$$
P\left\{\left|X_{n}\right|>t\right\} \leq P\{|X|>t\}, t \geq 0, n \geq 1 .
$$

Finally, a remark about notation is in order. Throughout, the symbol $\mathrm{C}$ denotes a generic constant $(0<\mathrm{C}<\infty)$ which is not necessarily the same one in each appearance.

\section{SLLN'S UNDER PROBABILISTIC AND GEOMETRIC CONDITIONS.}

With these preliminaries accounted for, the first group of results may be established. The random elements are assumed to be independent, and geometric conditions are placed on the real separable normed linear space. The space is assumed to be a Banach space of Rademacher type $p$ (for suitable $\mathrm{p}$ ) in Theorems 1-7, and it is assumed to be Beck-convex in Theorem 8 . The next lemma is the key lemma in establishing Theorems 1-4.

LEMMA 6. Let $\left(V_{n}, n \geq 1\right\}$ be independent random elements in a real separable, Rademacher type $p(1 \leq p \leq 2)$ Banach space $X$. Suppose that $\left\{V_{n}, n \geq 1\right\}$ is stochastically dominated by a ran- 
dom element $V$ in the sense that (1.2) holds. Let $\left\{a_{n}, n \geq 1\right\}$ and $\left\{b_{n}, n \geq 1\right\}$ be constants satisfying $0<b_{n} \uparrow \infty$ and

If

$$
\left(\max _{1 \leq j \leq n} \frac{b_{j}^{p}}{\left|a_{j}\right|^{p}}\right] \sum_{j=n}^{\infty} \frac{\left|a_{j}\right| p}{b_{j}^{p}}=O(n)
$$

$$
\sum_{n=1}^{\infty} P\left\{|| a_{n} V \mid I>D b_{n}\right\}<\infty
$$

then

$$
\frac{\sum_{j=1}^{n} a_{j}\left(V_{j}-E V_{j} I\left(|| a_{j} V_{j}|| \leq D^{2} b_{j}\right)\right)}{b_{n}} \rightarrow 0 \text { a.c. }
$$

PROOF. Let

$$
c_{n}=\frac{b_{n}}{\left|a_{n}\right|}, \quad Y_{n}=V_{n} I\left(|| V_{n}|| \leq D^{2} c_{n}\right), \quad n \geq 1
$$

Then for $\mathbf{n} \geq \mathbf{1}$

$$
\begin{array}{rl}
\sup _{m>n} & E|| \sum_{j=1}^{m} \frac{a_{j}\left(Y_{j}-E Y_{j}\right)}{b_{j}}-\sum_{j=1}^{n} \frac{a_{j}\left(Y_{j}-E Y_{j}\right)}{b_{j}} \| P \\
= & \sup _{m>n} E|| \sum_{j=n+1}^{m} \frac{a_{j}\left(Y_{j}-E Y_{j}\right)}{b_{j}}|| P \\
\left.\quad \leq \sup _{m>n} C \sum_{j=n+1}^{m} \frac{E|| Y_{j}-E Y_{j}|| P}{c_{j}^{p}} \text { (since } X \text { is of Rademacher type } p\right) \\
\quad \leq C 2^{p} \sum_{j=n+1}^{\infty} \frac{E|| Y_{j}|| P}{c_{j}^{p}}=o(1) \text { (by Lemma 2), }
\end{array}
$$

whence

$$
E|| \sum_{j=1}^{n} \frac{a_{j}\left(Y_{j}-E Y_{j}\right)}{b_{j}}-S|| P \rightarrow 0
$$

for some random element $S$ in $X$ implying

$$
\sum_{j=1}^{n} \frac{a_{j}\left(Y_{j}-E Y_{j}\right)}{b_{j}} \stackrel{P}{\rightarrow} S .
$$

Since convergence in probability and a.c. convergence are equivalent for sums of independent random elements in a separable Banach space (see Itô and Nisio [14]),

implying via the Kronecker lemma that

$$
\sum_{j=1}^{n} \frac{a_{j}\left(Y_{j}-E Y_{j}\right)}{b_{j}} \text { converges a.c. }
$$

$$
\frac{\sum_{j=1}^{n} a_{j}\left(Y_{j}-E Y_{j}\right)}{b_{n}} \rightarrow 0 \text { a.c. }
$$


However, $P\left\{\liminf _{n \rightarrow \infty}\left[V_{n}=Y_{n}\right]\right\}=1$ by the Borel-Cantelli lemma since (1.2) and (3.2) ensure that

$$
\left.\sum_{n=1}^{\infty} P\left(V_{n} \neq Y_{n}\right\}=\sum_{n=1}^{\infty} P\left\{|| V_{n} \|>D^{2} c_{n}\right\} \leq D \sum_{n=1}^{\infty} P|||V| \mid>D c_{n}\right\}<\infty .
$$

The conclusion (3.3) then follows directly from (3.5).

In the first theorem, there is a trade-off between the Rademacher type and the condition (3.6); the larger the Rademacher type $p$, the condition (3.6) becomes less stringent (since $b_{n} /\left|a_{n}\right| \uparrow$ ).

THEOREM 1. Let $\left\{V_{n}, n \geq 1\right\}$ be independent random elements in a real separable, Rademacher type $p(1 \leq p \leq 2)$ Banach space. Suppose that $\left\{V_{n}, n \geq 1\right\}$ is stochastically dominated by a random element $V$ in the sense that (1.2) holds. Let $\left\{a_{n}, n \geq 1\right\}$ and $\left\{b_{n}, n \geq 1\right\}$ bc constants satisfying $0<b_{n} \uparrow \infty, b_{n} /\left|a_{n}\right| \uparrow$,

$$
\frac{b_{n}^{p}}{\left|a_{n}\right|^{P}} \sum_{j=n}^{\infty} \frac{\left|a_{j}\right|^{P}}{b_{j}^{p}}=O(n),
$$

and

$$
\frac{b_{n}}{\left|a_{n}\right|} \sum_{j=1}^{n} \frac{\left|a_{j}\right|}{b_{j}}=O(n) .
$$

If the series of (3.2) converges, then the SLLN

$$
\frac{\sum_{j=1}^{n} a_{j}\left(V_{j}-E V_{j}\right)}{b_{n}} \rightarrow 0 \text { a.c. }
$$

obtains.

PROOF. Define $\left\{c_{n}, n \geq 1\right\}$ and $\left\{Y_{n}, n \geq 1\right\}$ as in (3.4). Note at the outset that (3.7) ensures that $c_{n} \leq C n, n \geq 1$, and so for all $j \geq 1$, by (1.2) and (3.2)

$$
\begin{aligned}
& \sum_{n=1}^{\infty} P\left\{|| V_{j}||>C D^{2} n\right\} \leq D \sum_{n=1}^{\infty} P\{|| V||>C D n\} \\
& \leq D \sum_{n=1}^{\infty} P\left\{|| V||>D c_{n}\right\}<\infty .
\end{aligned}
$$

Thus, E ||$V_{j} \|<\infty, j \geq 1$, and so (see, e.g., Taylor $[3, p .40]$ ) $V_{j}, j \geq 1$, all have expected values. Also, $c_{n} \uparrow \infty$ by (3.6).

Next, (3.3) holds by Lemma 6 and so it only needs to be demonstrated that

$$
\frac{\sum_{j=1}^{n} a_{j} E V_{j} I\left(|| V_{j}||>D^{2} c_{j}\right)}{b_{n}} \rightarrow 0 .
$$


To this end,

$$
\begin{aligned}
& \sum_{n=1}^{\infty} \frac{1}{c_{n}} E|| V_{n}|| I\left(|| V_{n}||>D^{2} c_{n}\right) \\
& \leq D^{2} \sum_{n=1}^{\infty} \frac{1}{c_{n}} E|| V|| I\left(|| V||>D c_{n}\right) \quad(b y(2.5)) \\
& =D^{2} \sum_{n=1}^{\infty} \frac{1}{c_{n}} \sum_{j=n}^{\infty} E|| V|| I\left(D c_{j}<|| V|| \leq D c_{j+1}\right) \\
& \leq D^{2} \sum_{j=1}^{\infty} E|| V|| I\left(D c_{j}<|| V|| \leq D c_{j+1}\right) \sum_{n=1}^{j+1} \frac{1}{c_{n}} \\
& \leq D^{3} \sum_{j=1}^{\infty} c_{j+1} P\left\{D c_{j}<|| V|| \leq D c_{j+1}\right) \frac{C(j+1)}{c_{j+1}} \quad(b y(3.7)) \\
& \leq C \sum_{j=1}^{\infty} j P\left\{D c_{j}<|| V|| \leq D c_{j+1}\right\} \\
& =C \sum_{j=1}^{\infty} \sum_{n=1}^{j} P\left\{D c_{j}<|| V|| \leq D c_{j+1}\right\} \\
& =C \sum_{n=1}^{\infty} \sum_{j=n}^{\infty} P\left\{D c_{j}<|| V|| \leq D c_{j+1}\right\} \\
& =C \sum_{n=1}^{\infty} P\left\{|| V||>D c_{n}\right\}<\infty \quad(b y(3.2))
\end{aligned}
$$

whence by the Kronecker lemma

$$
\frac{|| \sum_{j=1}^{n} a_{j} E V_{j} I\left(|| V_{j}||>D^{2} c_{j}\right)||}{b_{n}} \leq \frac{\sum_{j=1}^{n}\left|a_{j}\right| E|| V_{j}|| I\left(|| V_{j}||>D^{2} c_{j}\right)}{b_{n}}=o(1)
$$

REMARK. Apropos of Theorem 1, the authors are able to show through a slight modification of the argument that the condition $b_{n} /\left|a_{n}\right| \uparrow$ can be replaced by the somewhat weaker condition $b_{n} /\left|a_{n}\right|=O\left(\inf _{j \geq n} b_{j} /\left|a_{j}\right|\right)$.

THEOREM 2. Let $\left\{V_{n}, n \geq 1\right\}$ be independent random elements in a real separable, Rademacher type $p(1 \leq p \leq 2)$ Banach space. Suppose that $\left(V_{n}, n \geq 1\right)$ is stochastically dominated by a random element $V$ in the sense that $(1.2)$ holds, and suppose that $E|| V||<\infty$. Let $\left\{a_{n}, n \geq 1\right\}$ and $\left\{b_{n}, n \geq 1\right\}$ be constants satisfying $0<b_{n} \uparrow \infty,(3.1)$, and

$$
\sum_{j=1}^{n}\left|a_{j}\right|=O\left(b_{n}\right)
$$

If the series of (3.2) converges, then the SLLN

$$
\frac{\sum_{j=1}^{n} a_{j}\left(V_{j}-E V_{j}\right)}{b_{n}} \rightarrow 0 \text { a.c. }
$$

obtains. 
PROOF. Define $\left\{c_{n}, n \geq 1\right\}$ and $\left\{Y_{n}, n \geq 1\right\}$ as in (3.4). Note at the outset that (1.2) guarantees that $E|| V_{n}||<\infty, n \geq 1$, and so $V_{n}, n \geq 1$, all have expected values. Now (3.3) holds by Lemma 6 and so it only needs to be demonstrated that

$$
\frac{\sum_{j=1}^{n} a_{j} E V_{j} I\left(|| V_{j} \|>D^{2} c_{j}\right)}{b_{n}} \rightarrow 0 \text {. }
$$

To this end, note that (3.1) ensures $c_{n} \rightarrow \infty$, whence by (2.5), ElV\| $<\infty$, and the Lebesgue dominated convergence theorem

$$
\begin{aligned}
|| E V_{n} I\left(|| V_{n}||>D^{2} c_{n}\right) \| & \leq E|| V_{n}|| I\left(|| V_{n} \|>D^{2} c_{n}\right) \\
& \leq D^{2} E|| V|| I\left(|| V||>D c_{n}\right)=o(1) .
\end{aligned}
$$

But then by (3.8) and the Toeplitz lemma

$$
\frac{|| \sum_{j=1}^{n} a_{j} E V_{j} I\left(|| V_{j}||>D^{2} c_{j}\right)||}{b_{n}} \leq \frac{\sum_{j=1}^{n}\left|a_{j}\right||| E V_{j} I\left(|| V_{j} \|>D^{2} c_{j}\right)||}{b_{n}}=0(1) .
$$

THEOREM 3. Let $\left\{V_{n}, n \geq 1\right\}$ be independent random elements in a real separable, Rademacher type $p(1 \leq p \leq 2)$ Banach space. Suppose that $\left\{V_{n}, n \geq 1\right\}$ is stochastically dominated by a random element $\mathrm{V}$ in the sense that (1.2) holds, and suppose that

$$
\mathrm{P}(|| \mathrm{V}||>t) \text { is regularly varying with exponent } \rho<-1 \text {. }
$$

Let $\left\{a_{n}, n \geq 1\right\}$ and $\left\{b_{n}, n \geq 1\right\}$ be constants satisfying $0<b_{n} \uparrow \infty$ and (3.1). If the series of (3.2) converges, then the SLLN

$$
\frac{\sum_{j=1}^{n} a_{j}\left(V_{j}-E V_{j}\right)}{b_{n}} \rightarrow 0 \text { a.c. }
$$

obtains.

PROOF. Define $\left\{c_{n}, n \geq 1\right\}$ and $\left\{Y_{n}, n \geq 1\right\}$ as in (3.4). Now $E|| V||<\infty$ by Lemma 4 and so (1.2) ensures that $E|| V_{n}||<\infty, n \geq 1$, implying that $V_{n}, n \geq 1$, all have expected values. Again (3.3) holds by Lemma 6 and so it only needs to be demonstrated that

$$
\frac{\sum_{j=1}^{n} a_{j} E V_{j} I\left(|| V_{j}||>D^{2} c_{j}\right)}{b_{n}} \rightarrow 0 .
$$

To this end, it follows from (2.5), (3.1), and Lemma 4 that for all $n \geq$ some $n_{0}$

$$
\begin{aligned}
E|| V_{n}|| I\left(|| V_{n} \| \mid>D^{2} c_{n}\right) & \leq D^{2} E|| V|| I\left(|| V||>D c_{n}\right) \\
& \left.\leq C c_{n} P|||V| \mid>D c_{n}\right\} .
\end{aligned}
$$

Then by (3.2), 


$$
\sum_{n=1}^{\infty} \frac{1}{c_{n}} E|| V_{n}|| I\left(|| V_{n}||>D^{2} c_{n}\right) \leq C+C \sum_{n=n_{0}}^{\infty} P\left(|| V||>D c_{n} \mid<\infty\right.
$$

and so

$$
\frac{|| \sum_{j=1}^{n} a_{j} E V_{j} I\left(|| V_{j}||>D^{2} c_{j}\right)||}{b_{n}} \leq \frac{\sum_{j=1}^{n}\left|a_{j}\right| E|| V_{j}|| I\left(|| V_{j}||>D^{2} c_{j}\right)}{b_{n}}=o(1)
$$

by the Kronecker lemma.

REMARK. Apropos of Theorems 1, 2, or 3, Example 1 of Adler and Rosalsky [2] shows that the Theorems can fail without the assumption (3.7), (3.8), or (3.9), respectively.

The ensuing lemma can be helpful in verifying the conditions (3.6), (3.1), (4.6) of Theorems 1 , 2,3 , or 11 , and it will be used in the proof of Theorem 4 .

LEMMA 7 (Adler and Rosalsky [1]). Let $\left\{c_{n}, n \geq 1\right\}$ be constants with $0<c_{n}^{P / n} \uparrow$ for some $\mathrm{p}>0$. Then

$$
c_{n}^{p} \sum_{j=n}^{\infty} \frac{1}{c_{j}^{p}}=O(n)
$$

iff

$$
\liminf _{n \rightarrow \infty} \frac{c_{m}^{p}}{c_{n}^{p}}>r \text { for some integer } r \geq 2
$$

The next theorem is a random element version of a classical result of Feller [11] which had extended the Marcinkiewicz-Zygmund SLLN to more general norming constants.

THEOREM 4. Let $\left\{V_{n}, n \geq 1\right\}$ be i.i.d. random elements in a real separable, Rademacher type $p(1<p \leq 2)$ Banach space and let $\left\{b_{n}, n \geq 1\right\}$ be positive constants. Suppose that either

$$
\text { (i) } \mathrm{EV}_{1}=0, \quad \frac{\mathrm{b}_{\mathrm{n}}}{\mathrm{n}} \downarrow, \quad \frac{\mathrm{b}_{\mathrm{n}}}{\mathrm{n}^{\alpha}} \uparrow \quad \text { for some } \alpha>\frac{1}{\mathrm{p}}
$$

or

(ii) $E|| V_{1}||=\infty, \quad \frac{b_{n}}{n} \uparrow$.

If

$$
\sum_{n=1}^{\infty} P\left\{|| V_{1}||>b_{n}\right\}<\infty,
$$

then

$$
\frac{\sum_{j=1}^{n} v_{j}}{b_{n}} \rightarrow 0 \text { a.c. }
$$


PROOF. In either case $b_{n} \uparrow \infty$ and $b_{n}^{P} / n \uparrow$. Now $b_{n} / n^{\beta} \uparrow$ where $\beta=\alpha$ in case (i) and $\beta=1$ in case (ii). Thus,

$$
\liminf _{n \rightarrow \infty} \frac{b P_{n}}{b_{n}^{p}} \geq \liminf _{n \rightarrow \infty} \frac{(2 n)^{\beta p}}{n^{\beta p}}=2^{\beta p}>2,
$$

and so by Lemma 7

$$
b_{n}^{p} \sum_{j=n}^{\infty} \frac{1}{b_{j}^{p}}=O(n)
$$

Then by Lemma 6

$$
\frac{\sum_{j=1}^{n}\left(V_{j}-E V_{j} I\left(|| V_{j}|| \leq b_{j}\right)\right)}{b_{n}} \rightarrow 0 \text { a.c. }
$$

In case (i), $b_{n} / n \downarrow$ and (3.10) entail (see Chow and Teicher [10, pp. 123-124])

$$
\frac{|| \sum_{j=1}^{n} E V_{j} I\left(|| V_{j}||>b_{j}\right)||}{b_{n}} \leq \frac{\sum_{j=1}^{n} E|| V_{1}|| I\left(|| V_{1}||>b_{j}\right)}{b_{n}}=o(1)
$$

which when combined with (3.12) yields (3.11) since $\mathrm{EV}_{1}=0$.

In case (ii), in view of (3.10), necessarily $b_{n} / n \uparrow \infty$ and so (see Chow and Teicher [10, pp. 123-124])

$$
\frac{|| \sum_{j=1}^{n} E V_{j} I\left(|| V_{j} \| \leq b_{j}\right)||}{b_{n}} \leq \frac{\sum_{j=1}^{n} E|| V_{1}|| I\left(|| V_{1} \| \leq b_{j}\right)}{b_{n}}=0(1)
$$

yielding (3.11) via (3.12).

REMARK. In the special case where $\mathrm{EV}_{1}=0, \mathrm{E}|| \mathrm{V}_{1} \mid \|^{q}<\infty$ for some $1 \leq \mathrm{q}<\mathrm{p} \leq 2$, and $b_{n}=n^{1 / q}, n \geq 1$, Theorem 4(i) reduces to the Marcinkiewicz-Zygmund type SLLN $\sum_{j=1}^{n} V_{j} / n^{1 / q} \rightarrow 0$ a.c. of Woyczyński [15]. Woyczynski's result has been improved by de Acosta [16]. For some related results, see Wang and Bhaskara Rao [17].

THEOREM 5. Let $\left\{V_{n}, n \geq 1\right\}$ be independent random elements in a real separable, Rademacher type $p(1<p \leq 2)$ Banach space and suppose that

$$
\sup _{n \geq 1} E|| V_{n}||^{P}<\infty .
$$

Let $\left\{a_{n}, n \geq 1\right\}$ and $\left\{b_{n}, n \geq 1\right\}$ be constants such that $0<b_{n} \uparrow \infty$ and

Then the SLLN

$$
\frac{a_{n}}{b_{n}}=O\left(n^{-1 / p}(\log n)^{-1 / q}\right) \text { for some } 0<q<p
$$




$$
\frac{\sum_{j=1}^{n} a_{j}\left(V_{j}-E V_{j}\right)}{b_{n}} \rightarrow 0 \text { a.c. }
$$

obtains.

PROOF. Condition (3.13) ensures that $V_{n}, n \geq 1$, all have expected values. Let $c_{n}=b_{n} /\left|a_{n}\right|$, $Y_{n}=V_{n}\left(|| V_{n} \| \leq c_{n}\right), n \geq 1$. Now by (3.13) and (3.14)

$$
\sum_{n=1}^{\infty} \frac{E|| Y_{n}||^{P}}{c_{n}^{p}} \leq \sum_{n=1}^{\infty} \frac{E|| V_{n}|| P}{c_{n}^{p}} \leq C \sum_{n=1}^{\infty} \frac{1}{c_{n}^{p}}<\infty
$$

implying (see the proof of Lemma 6)

$$
\frac{\sum_{j=1}^{n} a_{j}\left(Y_{j}-E Y_{j}\right)}{b_{n}} \rightarrow 0 \text { a.c. }
$$

Now

$$
\sum_{n=1}^{\infty} P\left(V_{n} \neq Y_{n}\right\}=\sum_{n=1}^{\infty} P\left(|| V_{n} \|>c_{n}\right\} \leq \sum_{n=1}^{\infty} \frac{E\left\|\mid V_{n}\right\| P}{c_{n}^{P}}<\infty
$$

recalling (3.16), whence by the Borel-Cantelli lemma $P\left\{\lim \inf \left[V_{n}=Y_{n}\right]\right\}=1$ implying via (3.17) that

$$
\frac{\sum_{j=1}^{n} a_{j}\left(V_{j}-E Y_{j}\right)}{b_{n}} \rightarrow 0 \text { a.c. }
$$

Next,

$$
\begin{aligned}
\sum_{n=1}^{\infty} \frac{1}{c_{n}} & E|| V_{n}|| I\left(|| V_{n}||>c_{n}\right) \\
& =\sum_{n=1}^{\infty} P\left\{|| V_{n}||>c_{n} \mid+\sum_{n=1}^{\infty} \frac{1}{c_{n}} \int_{c_{n}}^{\infty} P\left\{|| V_{n}||>t\right\} d t \quad\right. \text { (by (2.4)) } \\
& \leq C+\sum_{n=1}^{\infty} \frac{1}{c_{n}} \int_{c_{n}}^{\infty} \frac{E|| V_{n}||^{P}}{t^{P}} d t \quad \text { (by (3.18)) } \\
& \leq C+C \sum_{n=1}^{\infty} \frac{1}{c_{n}^{P}}<\infty \quad \text { (by (3.13) and (3.16)), }
\end{aligned}
$$

and so by the Kronecker lemma

$$
\frac{|| \sum_{j=1}^{n} a_{j} E V_{j} I\left(|| V_{j}||>c_{j}\right)||}{b_{n}} \leq \frac{\sum_{j=1}^{n}\left|a_{j}\right| E|| V_{j}|| I\left(|| V_{j}||>c_{j}\right)}{b_{n}}=o(1)
$$

yielding (3.15) via (3.19).

THEOREM 6. Let $\left\{V_{n}, n \geq 1\right\}$ be independent random elements in a real separable, Rademacher type $p(1<p \leq 2)$ Banach space. Suppose that $\left\{V_{n}, n \geq 1\right\}$ is stochastically dominated by a random element $\mathrm{V}$ in the sense that (1.2) holds, and suppose that $\mathrm{E}|| \mathrm{V}||^{q}<\infty$ for some 
$1 \leq q<p$. Let $\left\{a_{n}, n \geq 1\right\}$ and $\left\{b_{n}, n \geq 1\right\}$ be constants satisfying $0<b_{n} \uparrow \infty$, (3.8), and

$$
\frac{a_{n}}{b_{n}}=O\left(n^{-1 / q}\right)
$$

Then the SLLN

obtains.

$$
\frac{\sum_{j=1}^{n} a_{j}\left(V_{j}-E V_{j}\right)}{b_{n}} \rightarrow 0 \text { a.c. }
$$

PROOF. Note that (1.2) entails $E|| V_{n}||^{q}<\infty, n \geq 1$, and hence $V_{n}, n \geq 1$, all have expected values. Let $c_{n}=b_{n} /\left|a_{n}\right|, Y_{n}=V_{n} I\left(|| V_{n}|| \leq n^{1 / q}\right), n \geq 1$. Now

$$
\begin{aligned}
& \sum_{n=1}^{\infty} \frac{E|| Y_{n}|| P}{c_{n}^{p}} \leq D \sum_{n=1}^{\infty} \frac{n^{p / q}}{c_{n}^{p}} P\left\{|| D V||>n^{1 / q}\right\} \\
& +D^{p+1} \sum_{n=1}^{\infty} \frac{1}{c_{n}^{p}} E|| V|| P I\left(|| D V|| \leq n^{1 / q}\right) \quad(\text { by }(2.3)) \\
& \leq C+C \sum_{n=1}^{\infty} n^{-p / q} \sum_{k=1}^{n} E|| V||^{P} I\left((k-1)^{1 / q}<|| D V|| \leq k^{1 / q}\right) \\
& \text { (by } \left.(3.20) \text { and } E|| V||^{q}<\infty\right) \\
& =\mathrm{C}+\mathrm{C} \sum_{\mathbf{k}=1}^{\infty} \mathrm{E}|| \mathrm{V}|| \mathrm{PI}\left((\mathrm{k}-1)^{1 / q}<|| D V|| \leq \mathrm{k}^{1 / q}\right) \sum_{\mathbf{n}=\mathbf{k}}^{\infty} \mathrm{n}^{-\mathrm{p} / \mathrm{q}} \\
& \left.\leq \mathrm{C}+\mathrm{C} \sum_{k=1}^{\infty} k^{(\mathrm{q}-\mathrm{p}) / \mathrm{q}} \mathrm{E}|| \mathrm{V}||^{\mathrm{P} I\left((k-1)^{1 / q}<|| D V \|\right.} \mid \leq \mathrm{k}^{1 / \mathrm{q}}\right) \\
& \leq \mathrm{C}+\mathrm{C} \sum_{\mathrm{k}=1}^{\infty} \mathrm{E}|| \mathrm{V}|| \mathrm{q} I\left((\mathrm{k}-1)^{1 / \mathrm{q}}<\left\||\mathrm{DV} \|| \leq \mathrm{k}^{1 / \mathrm{q}}\right)\right. \\
& =\mathrm{C}+\mathrm{CE}\|\| \mathrm{V}\|\|^{9}<\infty
\end{aligned}
$$

implying (see the proof of Lemma 6)

$$
\frac{\sum_{j=1}^{n} a_{j}\left(Y_{j}-E Y_{j}\right)}{b_{n}} \rightarrow 0 \text { a.c. }
$$

Now by $(1.2)$ and $E|| V||^{9}<\infty$,

$$
\sum_{n=1}^{\infty} P\left\{V_{n} \neq Y_{n}\right\}=\sum_{n=1}^{\infty} P\left\{|| V_{n}||>n^{1 / q}\right\} \leq D \sum_{n=1}^{\infty} P\left\{|| D V||>n^{1 / q}\right\}<\infty,
$$

and so by the Borel-Cantelli lemma $P\left\{\liminf _{n \rightarrow \infty}\left[V_{n}=Y_{n}\right]\right\}=1$ implying via (3.22) that

$$
\frac{\sum_{j=1}^{n} a_{j}\left(V_{j}-E Y_{j}\right)}{b_{n}} \rightarrow 0 \text { a.c. }
$$

Next, by (2.5), ElV $\|<\infty$, and the Lebesgue dominated convergence theorem

$$
E|| V_{n}|| I\left(|| V_{n}||>n^{1 / q}\right) \leq D^{2} E|| V|| I\left(|| D V||>n^{1 / q}\right)=o(1),
$$

whence by (3.8) and the Toeplitz lemma 


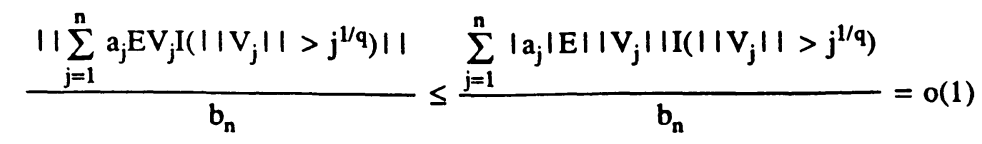

yielding (3.21) via (3.23).

The following Corollary is an extension of Theorem 2 of Adler and Rosalsky [2] (which, in tum, is an extension of Theorem 3.1 of Fernholz and Teicher [18]) and establishes a SLLN for normed weighted sums of stochastically dominated random variables. It will be used in the proofs of Theorems 8 and 9 but may be of independent interest.

COROLLARY 1. Let $\left\{X_{n}, n \geq 1\right\}$ be independent random variables and let $X$ be an $L_{p}$ random variable for some $1 \leq p<2$. Suppose that $\left\{X_{n}, n \geq 1\right\}$ is stochastically dominated by $X$ in the sense that there exists a constant $D<\infty$ such that

$$
P\left(\left|X_{n}\right|>t\right) \leq D P(|D X|>t), \quad t \geq 0, n \geq 1 .
$$

Let $\left\{a_{n}, n \geq 1\right\}$ and $\left\{b_{n}, n \geq 1\right\}$ be constants satisfying $0<b_{n} \uparrow \infty, a_{n} / b_{n}=O\left(n^{-1 / P}\right)$, and (3.8).

Then the SLLN

$$
\frac{\sum_{j=1}^{n} a_{j}\left(X_{j}-E X_{j}\right)}{b_{n}} \rightarrow 0 \text { a.c. }
$$

obtains.

PROOF. Since $(\mathrm{R},|\cdot|)$ is a real separable, Rademacher type 2 Banach space, the Corollary follows immediately from Theorem 6 with $p=2$ and $q=p<2$.

THEOREM 7. Let $\left\{V_{n}, n \geq 1\right\}$ be independent random elements in a real separable, Rademacher type $p(1 \leq p \leq 2)$ Banach space. Suppose that $\left\{V_{n}, n \geq 1\right\}$ is stochastically dominated by a random element $\mathrm{V}$ in the sense that (1.2) holds, and suppose that $\mathrm{E}|| \mathrm{V}||^{\mathrm{P}}<\infty$. Let $\left\{a_{n}, n \geq 1\right\}$ and $\left\{b_{n}, n \geq 1\right\}$ be constants satisfying $0<b_{n} \uparrow \infty,(3.8)$, and (3.14). Then the SLLN

obtains.

$$
\frac{\sum_{j=1}^{n} a_{j}\left(V_{j}-E V_{j}\right)}{b_{n}} \rightarrow 0 \text { a.c. }
$$

PROOF. Using the truncation $Y_{n}=V_{n} I\left(|| V_{n}|| \leq n^{1 / P}\right), n \geq 1$, the argument is a slight madification of that used to establish Theorem 6. The details are left to the reader.

REMARK. An interesting question which we are unable to resolve is whether Theorem 7 holds with (3.14) replaced by the somewhat weaker condition $a_{n} / b_{n}=O\left(n^{-1 / p}\right)$. Moreover, Theorem 
7 should be compared with Theorem 10 wherein the $\left(V_{n}, n \geq 1\right\}$ are (uniformly) tight.

The next Theorem establishes a SLLN for normed weighted sums of random elements in a real separable normed linear space which is Beck-convex. It should be compared with Theorem 5 of Taylor and Padgett [19] (or Theorem 5.3.1 of Taylor [3, p. 137]).

THEOREM 8. Let $\left\{V_{n}, n \geq 1\right\}$ be independent random elements in a real separable normed linear space which is Beck-convex and let $\left\{a_{n}, n \geq 1\right\}$ and $\left\{b_{n}, n \geq 1\right\}$ be constants satisfying $a_{n}>0, n \geq 1,0<b_{n} \uparrow \infty, \sum_{j=1}^{n} a_{j}=O\left(b_{n}\right), a_{n} / b_{n}=O\left(n^{-1 / p}\right)$ for some $1 \leq p<2$, and

$$
\sum_{j=1}^{n}\left(a_{j}-d_{n}\right)=o\left(b_{n}\right)
$$

where $d_{n} \equiv \min _{1 \leq j \leq n} a_{j}, n \geq 1$. If $\sup _{n \geq 1} E|| V_{n}||^{q}<\infty$ for some $q>p$, then the SLLN

$$
\frac{\sum_{j=1}^{n} a_{j}\left(V_{j}-E V_{j}\right)}{b_{n}} \rightarrow 0 \text { a.c. }
$$

obtains.

PROOF. Without loss of generality, it may and will be supposed that $\mathrm{EV}_{\mathbf{n}}=0, \mathrm{n} \geq 1$. Suppose, initially, that the $\left\{V_{n}, n \geq 1\right\}$ are uniformly bounded in the norm by a constant, that is, $\sup _{n \geq 1}|| V_{n}|| \leq C$ a.c. Then, since $n d_{n} \leq \sum_{j=1}^{n} a_{j}=O\left(b_{n}\right)$,

$$
\begin{aligned}
\frac{|| \sum_{j=1}^{n} a_{j} V_{j} \|}{b_{n}} & \leq \frac{|| \sum_{j=1}^{n}\left(a_{j}-d_{n}\right) V_{j}||}{b_{n}}+\frac{d_{n}|| \sum_{j=1}^{n} V_{j} \|}{b_{n}} \\
& \leq \frac{C \sum_{j=1}^{n}\left(a_{j}-d_{n}\right)}{b_{n}}+\frac{C|| \sum_{j=1}^{n} V_{j}||}{n} \rightarrow 0 \text { a.c. }
\end{aligned}
$$

by (3.24) and a SLLN of Beck [20, Theorem 10] (which is Theorem 4.3.1 of Taylor [3, p. 87]) thereby proving the theorem when $\sup _{n \geq 1}|| V_{n} \| \leq C$ a.c.

Next, in general, define

$$
X_{n}=V_{n} I\left(|| V_{n} \| \leq M\right), \quad Y_{n}=V_{n} I\left(|| V_{n} \|>M\right), \quad n \geq 1,
$$

where $1<M<\infty$ is a constant. By the portion of the theorem already proved,

$$
\frac{\sum_{j=1}^{n} a_{j}\left(X_{j}-E X_{j}\right)}{b_{n}} \rightarrow 0 \text { a.c. }
$$

Note that for $\mathrm{n} \geq 1$, 


$$
\begin{aligned}
E|| Y_{n}|| & =\frac{E\left\{M^{q-1}|| V_{n}|| I\left(|| V_{n}||>M\right)\right\}}{M^{q-1}} \\
& \leq \frac{E|| V_{n}|| q I\left(|| V_{n}||>M\right)}{M^{q-1}} \leq \frac{C}{M^{q-1}}
\end{aligned}
$$

and so in view of $\sum_{j=1}^{n} a_{j}=O\left(b_{n}\right)$

$$
\begin{aligned}
\frac{\| \sum_{j=1}^{n} a_{j}\left(Y_{j}-E Y_{j}\right)||}{b_{n}} & \leq \frac{\sum_{j=1}^{n} a_{j}|| Y_{j}||}{b_{n}}+\frac{\sum_{j=1}^{n} a_{j} E|| Y_{j}||}{b_{n}} \\
& =\frac{\sum_{j=1}^{n} a_{j}\left(|| Y_{j}||-E|| Y_{j}||\right)}{b_{n}}+\frac{2 \sum_{j=1}^{n} a_{j} E|| Y_{j}||}{b_{n}} \\
& \leq \frac{\sum_{j=1}^{n} a_{j}\left(|| Y_{j}||-E|| Y_{j}||\right)}{b_{n}}+\frac{C}{M^{q-1}} .
\end{aligned}
$$

Now $\left\{|| Y_{n}||-E|| Y_{n}||, n \geq 1\right\}$ are independent mean 0 random variables with

$$
\sup _{n \geq 1} E||\left|Y_{n}\right||-E|\left|Y_{n}\right|||^{q} \leq 2^{q} \sup _{n \geq 1} E|| Y_{n}||^{q} \leq 2^{q} \sup _{n \geq 1} E|| V_{n}||^{q}<\infty .
$$

By Lemma 5, there exists a random variable $\mathrm{Y}$ with $\mathrm{E} /\left.\mathrm{Y}\right|^{\mathrm{P}}<\infty$ such that

$$
P\left\{||\left|Y_{n}\right||-E|\left|Y_{n}\right|||>t\right\} \leq P\{|Y|>t\}, \quad t \geq 0, n \geq 1,
$$

whence by Corollary 1

But then by (3.26) and (3.27)

$$
\frac{\sum_{j=1}^{n} a_{j}\left(|| Y_{j}||-E|| Y_{j}||\right)}{b_{n}} \rightarrow 0 \text { a.c. }
$$

$$
\begin{aligned}
\limsup _{n \rightarrow \infty} \frac{\left\|\sum_{j=1}^{n} a_{j} V_{j}\right\|}{b_{n}} & \leq \limsup _{n \rightarrow \infty} \frac{\| \sum_{j=1}^{n} a_{j}\left(X_{j}-E X_{j}\right)||}{b_{n}} \\
& +\limsup _{n \rightarrow \infty} \frac{\| \sum_{j=1}^{n} a_{j}\left(Y_{j}-E Y_{j}\right)||}{b_{n}} \\
& \leq \frac{C}{M^{q-1}} \text { a.c. }
\end{aligned}
$$

and since $M$ is arbitrary, the conclusion (3.25) follows.

\section{SLLN'S UNDER PROBABILISTIC CONDITIONS.}

In this section, SLLN's are obtained without imposing geometric conditions on the Banach space. As in Section 3, moment conditions are placed on independent random elements and restrictions are placed on the constants $\left\{a_{n}, n \geq 1\right\}$ and $\left\{b_{n}, n \geq 1\right\}$. In Theorem 9, the Banach space is assumed to admit a Schauder basis and in Theorem 10, the independent random elements in a 
Banach space are assumed to be (uniformly) tight.

For a Banach space admitting a Schauder basis, recall the definitions of $\left(f_{i}, i \geq 1\right)$, $\left\{U_{m}, m \geq 1\right\}$, and $\left\{Q_{m}, m \geq 1\right\}$ presented in Section 2. Theorem 9 should be compared with Theorem 5.1.4 of Taylor [3, p.114].

THEOREM 9. Let $\left\{V_{n}, n \geq 1\right\}$ be independent, mean zero random elements in a real separable Banach space admitting a Schauder basis $\left\{\beta_{i}, i \geq 1\right\}$. Let $\left\{a_{n}, n \geq 1\right\}$ and $\left\{b_{n}, n \geq 1\right\}$ be constants satisfying $0<b_{n} \uparrow \infty,(3.8)$, and

$$
\frac{a_{n}}{b_{n}}=O\left(n^{-1 / p}\right)
$$

for some $1 \leq p<2$. Suppose that there exist random variables $\left\{X_{i}, i \geq 1\right\}$ and $\left(Y_{m}, m \geq 1\right\}$ and a constant $\mathrm{D}<\infty$ such that

$$
\begin{aligned}
& P\left(\left|f_{i}\left(V_{n}\right)\right|>t\right\} \leq D P\left(\left|D X_{i}\right|>t\right), \quad t \geq 0, n \geq 1, i \geq 1, \\
& \left.P\left(||\left|Q_{m}\left(V_{n}\right)\right| 1-E|| Q_{m}\left(V_{n}\right)|| \mid>t\right\} \leq D P|| D Y_{m} \mid>t\right\}, \quad t \geq 0, m \geq 1, n \geq 1, \\
& \sup _{i \geq 1} E\left|X_{i}\right| P<\infty, \quad \sup _{m \geq 1} E\left|Y_{m}\right| P<\infty,
\end{aligned}
$$

and

Then the SLLN

$$
\lim _{m \rightarrow \infty} \sup _{n \geq 1} E|| Q_{m}\left(V_{n}\right)||=0
$$

obtains.

$$
\frac{\sum_{j=1}^{n} a_{j} V_{j}}{b_{n}} \rightarrow 0 \text { a.c. }
$$

PROOF. It follows directly from Corollary 1 that

$$
\frac{\sum_{j=1}^{n} a_{j} f_{i}\left(V_{j}\right)}{b_{n}} \rightarrow 0 \text { a.c. } \quad \text { for each } i \geq 1
$$

and

$$
T_{m, n} \equiv \frac{\sum_{j=1}^{n}\left|a_{j}\right|\left(|| Q_{m}\left(V_{j}\right)||-E|| Q_{m}\left(V_{j}\right) \mid l\right)}{b_{n}} \rightarrow 0 \text { a.c. for each } m \geq 1
$$

Then

$$
\begin{aligned}
\left\|U_{m}\left[\frac{\sum_{j=1}^{n} a_{j} V_{j}}{b_{n}}\right]\right\| & =\left\|\sum_{i=1}^{m} f_{i}\left(\frac{\sum_{j=1}^{n} a_{j} V_{j}}{b_{n}}\right] \beta_{i}\right\| \\
& \leq \sum_{i=1}^{m}\left|\frac{\sum_{j=1}^{n} a_{j} f_{i}\left(V_{j}\right)}{b_{n}}\right||| \beta_{i} \| \rightarrow 0 \text { a.c. for each } m \geq 1 .
\end{aligned}
$$


Thus, by (4.4), (4.3), (3.8), and (4.2)

$$
\begin{aligned}
\frac{\left\|\sum_{j=1}^{n} a_{j} V_{j}\right\|}{b_{n}} & \leq\left\|U_{m}\left(\frac{\sum_{j=1}^{n} a_{j} V_{j}}{b_{n}}\right)\right\|+\frac{\left\|\sum_{j=1}^{n} a_{j} Q_{m}\left(V_{j}\right)\right\|}{b_{n}} \\
& \leq\left\|U_{m}\left[\frac{\sum_{j=1}^{n} a_{j} V_{j}}{b_{n}}\right]\right\|+T_{m, n}+\underset{R}{C} \operatorname{csup}_{R}|| Q_{m}\left(V_{j}\right) \| \\
& \rightarrow 0 \text { a.c. as first } n \rightarrow \infty \text { and then } m \rightarrow \infty . \square
\end{aligned}
$$

THEOREM 10. Let $\left\{V_{n}, n \geq 1\right\}$ be a (uniformly) tight sequence of independent, mean zero random elements in a real separable Banach space $X$. Let $\left\{a_{n}, n \geq 1\right\}$ and $\left\{b_{n}, n \geq 1\right\}$ be constants satisfying $0<b_{n} \uparrow \infty,(3.8)$, and (4.1) for some $1 \leq p<2$. Suppose that $\left(V_{n}, n \geq 1\right\}$ is stochastically dominated by a random element $\mathrm{V}$ in the sense that (1.2) holds, and suppose that EI|V||P $<\infty$. Then the SLLN

$$
\frac{\sum_{j=1}^{n} a_{j} v_{j}}{b_{n}} \rightarrow 0 \text { a.c. }
$$

obtains.

PROOF. Let $h$ be a norm-preserving, bicontinuous, linear mapping of $X$ into $c[0,1](=$ the Banach space of all continuous real-valued functions y on $[0,1]$ with norm ||$\left.y||=\sup _{0 \leq 1 \leq 1}|y(t)|\right)$. The Banach space $c[0,1]$ admits a monotone basis where ||$Q_{m}(y)\|\leq\| y \|$ and $\left|f_{m}(y)\right| \leq|| y||$ for each $y \in C[0,1]$ and $m \geq 1$ and where $\left\{\left\|Q_{m}(y)\right\|, m \geq 1\right\}$ is a monotone decreasing sequence for each $y \in C[0,1]$. Then $\left\{h\left(V_{n}\right), n \geq 1\right\}$ is a (uniformly) tight sequence of independent, mean zero random elements in $c[0,1]$. Now for arbitrary $\varepsilon>0$, choose $u>0$ so that $D^{2} E|| V|| I(|| V||>u) \leq \frac{\varepsilon}{3}$. Then Lemma 3 provides $E|| V_{n}|| I\left(|| V_{n} \|>u\right) \leq \frac{\varepsilon}{3}$ for all $n \geq 1$. By (uniform) tightness, a compact subset $K$ of $c[0,1]$ may be chosen so that $P\left(h\left(V_{n}\right) \notin K\right\} \leq \frac{\varepsilon}{3 u}$ for all $n \geq 1$, whence $E|| V_{n}|| I\left(|| V_{n} \| \leq u\right) I\left(h\left(V_{n}\right) \notin K\right) \leq \frac{\varepsilon}{3}$ for all $n \geq 1$. Since ||$Q_{m}(y)|| \downarrow 0$ for each $y$ in the compact set $K$, Dini's theorem ensures that there exists an integer $m_{0}$ such that $\sup _{y \in K}|| Q_{m}(y)|| \leq \frac{\varepsilon}{3}$ for all $m \geq m_{0}$. Then for all $m \geq m_{0}$ and $n \geq 1$ $E|| Q_{m}\left(h\left(V_{n}\right)\right)|| \leq E|| Q_{m}\left(h\left(V_{n}\right)\right) I\left(|| V_{n}|| \leq u\right) I\left(h\left(V_{n}\right) \in K\right)||$

$$
+E|| V_{n}|| I\left(|| V_{n} \| \leq u\right) I\left(h\left(V_{n}\right) \notin K\right)+E \|\left|V_{n}\right| \mid I\left(|| V_{n} \|>u\right) \leq \varepsilon
$$


thereby establishing (4.2) for the random elements $\left\{h\left(V_{n}\right), n \geq 1\right\}$. The identifications $X_{i}=|| V||$ and $Y_{m}=\||V||+D E||V| \mid$ for all $i \geq 1$ and $m \geq 1$ ensure that the other conditions of Theorem 9 hold. Thus

$$
\frac{|| \sum_{j=1}^{n} a_{j} V_{j} \|}{b_{n}}=\frac{|| h\left(\sum_{j=1}^{n} a_{j} V_{j}\right) \| \mid}{b_{n}}=\frac{\| \sum_{j=1}^{n} a_{j} h\left(V_{j}\right)||}{b_{n}} \rightarrow 0 \text { a.c. }
$$

REMARKS. (i) When $a_{n}=1, b_{n}=n, n \geq 1$, and $\sup _{n \geq 1} E|| V_{n}|| p<\infty$ for some $p>1$, Theorem 10 in conjunction with Lemma 5 will establish the SLLN of Taylor [3, Corollary 5.2.9, p. 133]. As pointed out by Taylor [3, p. 133], that same SLLN can be obtained from Theorem 5.2.8 of Taylor $\left[3\right.$, p. 131] but under the stronger assumption that $\sup _{n \geq 1} E|| V_{n}|| P<\infty$ for some $p>2$.

(ii) Theorem 10 can fail if $p=1$ and $E|| V||=\infty$. For an example, see Taylor [3, Example 5.2.3, p. 135 ].

The next Corollary should be compared with Theorem 5.2.8 of Taylor [3, p. 131].

COROLLARY 2. Let $\left(V_{n}, n \geq 1\right\}$ be a (uniformly) tight sequence of independent, mean zero random elements in a real separable Banach space. Let $\left\{a_{n}, n \geq 1\right\}$ and $\left\{b_{n}, n \geq 1\right\}$ be constants satisfying $0<b_{n} \uparrow \infty,(3.8)$, and (4.1) for some $1 \leq p<2$. If

$$
\sup _{n \geq 1} E|| V_{n}||^{q}<\infty \text { for some } q>p \text {, }
$$

then the SLLN

$$
\frac{\sum_{j=1}^{n} a_{j} V_{j}}{b_{n}} \rightarrow 0 \text { a.c. }
$$

obtains.

PROOF. Condition (4.5) ensures by Lemma 5 that (1.2) obtains and $E|| V||^{P}<\infty$. The Corollary then follows from Theorem 10.

In the next Corollary, the sequence $\left(V_{n}, n \geq 1\right\}$ is i.i.d. and the moment condition (4.5) is weakened to $E|| V_{1}|| P$. The Corollary should be compared with Theorem 5.1.3 of Taylor $[3$, p. 112].

COROLLARY 3. Let $\left(V_{n}, n \geq 1\right\}$ be i.i.d. mean zero random elements in a real separable Banach space. Let $\left(a_{n}, n \geq 1\right\}$ and $\left\{b_{n}, n \geq 1\right\}$ be constants satisfying $0<b_{n} \uparrow \infty$, (3.8), and (4.1) for some $1 \leq p<2$. If $E|| V_{1}||^{p}<\infty$, then the SLLN 


$$
\frac{\sum_{j=1}^{n} a_{j} v_{j}}{b_{n}} \rightarrow 0 \text { a.c. }
$$

obtains.

PROOF. Since the i.i.d. hypothesis ensures that $\left(V_{n}, n \geq 1\right\}$ is automatically (uniformly) tight (see Taylor [3, p. 121]), the Corollary follows immediately from Theorem 10.

REMARKS. (i) In the particular case where $a_{n} \equiv 1, b_{n} \equiv n$, and $p=1$, Corollary 3 reduces to the SLLN of Mourier [7].

(ii) A Fréchet space is a complete linear metric space. Using Theorem 10, a SLLN may be obtained for random elements in a real separable Fréchet space $F$ which is a locally convex space with a countable family of seminorms $\left\{\rho_{k}, k \geq 1\right\}$ defined on it such that the metric $d$ is defined by

$$
d(x, y)=\sum_{k=1}^{\infty} \frac{\rho_{k}(x-y)}{2^{k}\left(1+\rho_{k}(x-y)\right)} \quad \text { for } x, y \in F .
$$

The details will not be given since the argument parallels that of Theorem 5.2.10 of Taylor [3,p. 136]. (Corollary 2 plays the same role in the proof as Theorem 5.2 .8 of Taylor $[3$, p. 131] played in proving Theorem 5.2.10.) In fact, almost all of the results in this section have parallel results for Fréchet spaces.

In the last theorem, there is no independence assumption on the sequence of random elements. Moreover, the space is equipped with a seminorm $\rho$ which is not necessarily a norm and thus the result is applicable to a larger class of spaces than real separable normed linear spaces. The definition of random element is analogous to that discussed in Section 1 for real separable normed linear spaces.

THEOREM 11. Let $\left\{V_{n}, n \geq 1\right\}$ be random elements in a real separable seminormed linear space with seminorm $\rho$. Suppose that $\left\{V_{n}, n \geq 1\right\}$ is stochastically dominated by a random element $\mathrm{V}$ in the sense that there exists a constant $\mathrm{D}<\infty$ such that

$$
P\left(\rho\left(V_{n}\right)>t\right) \leq D P\{\rho(D V)>t\}, \quad t \geq 0, n \geq 1 .
$$

Let $\left\{a_{n}, n \geq 1\right\}$ and $\left\{b_{n}, n \geq 1\right\}$ be constants such that $0<b_{n} \uparrow \infty$ and

$$
\left[\max _{1 \leq j \leq n} \frac{b_{j}}{\left|a_{j}\right|}\right]_{j=n}^{\infty} \frac{\left|a_{j}\right|}{b_{j}}=O(n) .
$$


If

$$
\sum_{n=1}^{\infty} P\left(\rho\left(a_{n} V\right)>D b_{n}\right)<\infty
$$

then

$$
\frac{\rho\left[\sum_{j=1}^{n} a_{j} V_{j}\right)}{b_{n}} \rightarrow 0 \text { a.c. }
$$

PROOF. Set $Y_{n}=\rho\left(V_{n}\right), n \geq 1$, and $Y=\rho(V)$. Then by Theorem 2 of Adler and Rosalsky [1],

$$
\frac{\rho\left[\sum_{j=1}^{n} a_{j} V_{j}\right]}{b_{n}} \leq \frac{\sum_{j=1}^{n}\left|a_{j}\right| \rho\left(V_{j}\right)}{b_{n}} \rightarrow 0 \text { a.c. }
$$

\section{REFERENCES}

1. ADLER, A. and ROSALSKY, A. Some General Strong Laws for Weighted Sums of Stochastically Dominated Random Variables, Stochastic Anal. Appl. 5 (1987), 1-16.

2. ADLER, A. and ROSALSKY, A. On the Strong Law of Large Numbers for Normed Weighted Sums of I.I.D. Random Variables, Stochastic Anal. Appl. 5 (1987), 467-483.

3. TAYLOR, R.L. Stochastic Convergence of Weighted Sums of Random Elements in Linear Spaces, Lecture Notes in Math. 672. Springer-Verlag, Berlin 1978.

4. ROHATGI, V.K. Convergence of Weighted Sums of Independent Random Variables, Proc. Cambridge Philos. Soc. 69 (1971), 305-307.

5. PRUITT, W.E. Summability of Independent Random Variables, J. Math. Mech. 15 (1966), 769-776.

6. MIKOSCH, T. and NORVAIŠA, R. On Almost Sure Behavior of Sums of Independent Banach Space Valued Random Variables, Math. Nachr. 125 (1986), 217-231.

7. MOURIER, E. Eléments Aléatoires dans un Espace de Banach, Ann. Inst. Henri Poincaré Sect. B, 13 (1953), 159-244.

8. LAHA, R.G. and ROHATGI, V.K. Probability Theory John Wiley, New York, 1979.

9. CUESTA, J.A. and MATRAN, C. Strong Laws of Large Numbers in Abstract Spaces via Skorohod's Representation Theorem, Sankhyā. Ser. A, $\underline{48}$ (1986), 98-103.

10. CHOW, Y.S. and TEICHER, H. Probability Theory: Independence, Interchangeability.Martingales. Springer-Verlag, New York, 1978.

11. FELLER, W. A Limit Theorem for Random Variables with Infinite Moments, Amer. J. Math. $\underline{68}$ (1946), 257-262.

12. HOFFMANN-JGRGENSEN, J. and PISIER, G. The Law of Large Numbers and the Central Limit Theorem in Banach Spaces, Ann. Probab. 4 (1976), 587-599.

13. GIESY, D.P. On a Convexity Condition in Normed Linear Spaces, Trans. Amer. Math. Soc. $\underline{125}$ (1966), 114-146.

14. ITÔ, K. and NISIO, M. On the Convergence of Sums of Independent Banach Space Valued Random Variables, Osaka J. Math. 5 (1968), 35-48. 
15. WOYCZYNSKI, W.A. On Marcinkiewicz-Zygmund Laws of Large Numbers in Banach Spaces and Related Rates of Convergence, Probab. and Math. Statist. 1 (1980), 117-131.

16. DE ACOSTA, A. Inequalities for B-Valued Random Vectors with Applications to the Strong Law of Large Numbers, Ann. Probab. 9 (1981), 157-161.

17. WANG, X.C. and BHASKARA RAO, M. Some Results on the Convergence of Weighted Sums of Random Elements in Separable Banach Spaces, Studia Math. 86 (1987), 131-153.

18. FERNHOLZ, L.T. and TEICHER, H. Stability of Random Variables and Iterated Logarithm Laws for Martingales and Quadratic Forms, Ann. Probab. 8 (1980), 765-774.

19. TAYLOR, R.L. and PADGETT, W.J. Stochastic Convergence of Weighted Sums in Normed Linear Spaces, J. Multivariate Anal. 5 (1975), 434-450.

20. BECK, A. On the Strong Law of Large Numbers, In Ergodic Theory; Proceedings of an International Symposium Held at Tulane University. New Orleans, Louisiana, October, 1961 (Ed. F.B. Wright), 21-53, Academic Press, New York, 1963. 


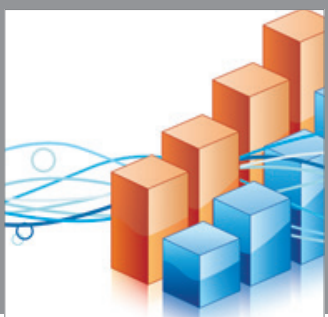

Advances in

Operations Research

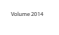

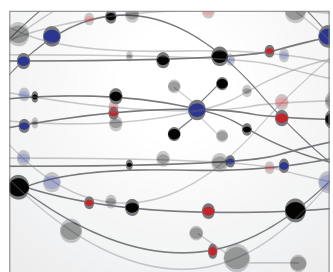

\section{The Scientific} World Journal
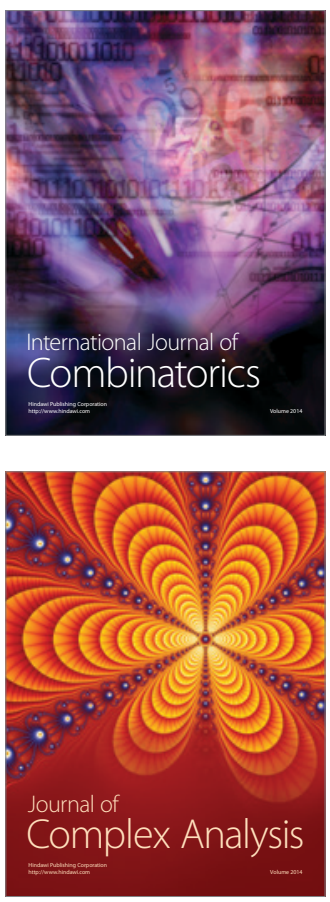

International Journal of

Mathematics and

Mathematical

Sciences
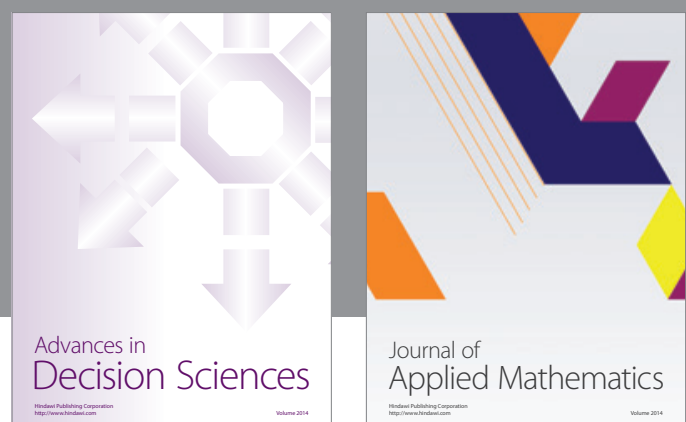

Journal of

Applied Mathematics
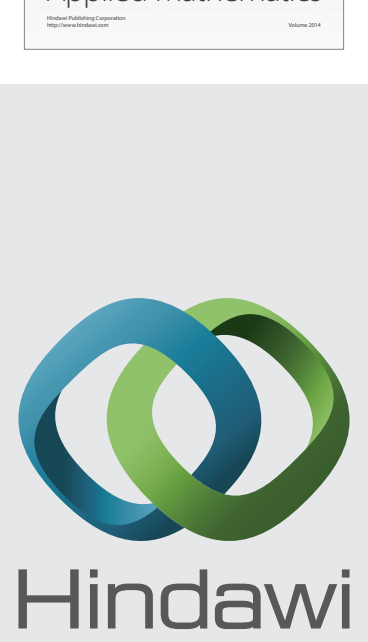

Submit your manuscripts at http://www.hindawi.com
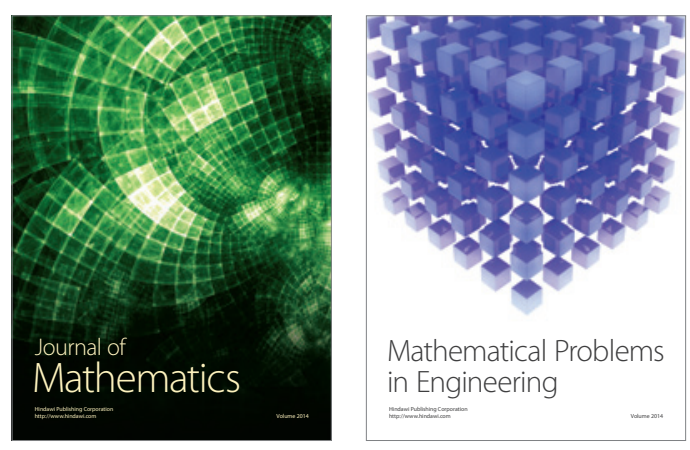

Mathematical Problems in Engineering
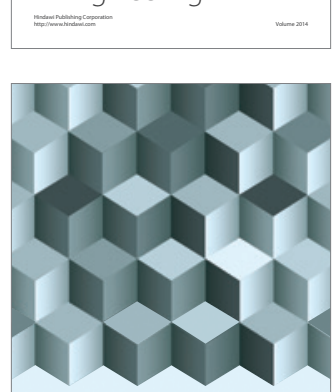

Journal of

Function Spaces
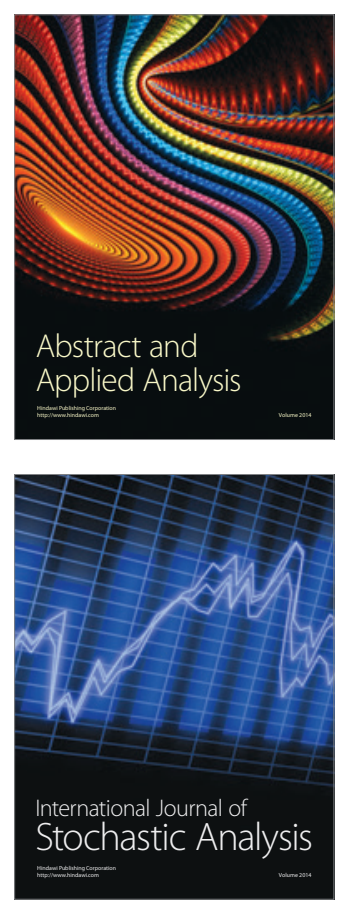

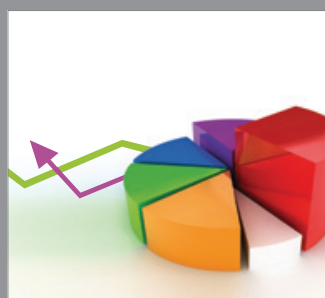

ournal of

Probability and Statistics

Promensencen
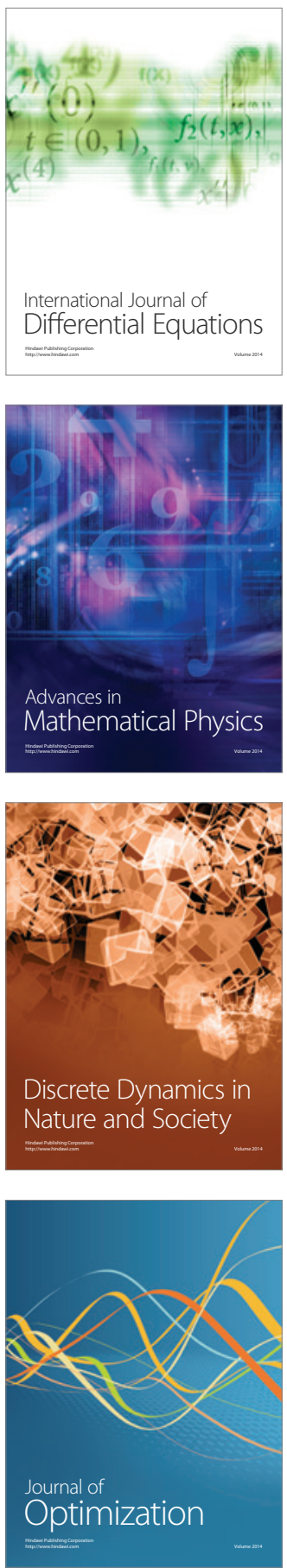